\title{
A Competent and Readily Understandable Book on Radiation for a Wide Range of Readers
}

PACS numbers: $01.30 . \mathrm{Vv}$

DOI: $10.1134 / \mathrm{S} 1066362209040237$

It is well known that radiation problems in Russia, as well as in other countries, arouse interest of a wide range of readers, including students. Seemingly, there should not be any shortage of such literature. However, this is not the case. Books appearing in book shops either are too complex for understanding by unprepared readers or have numerous inaccuracies and even bad mistakes. A book of small volume, written by noted Russian radiochemist Yu.V. Egorov [Egorov, Yu.V., Radiatsiya kak biosfernyi faktor: kurs lektsii (Radiation as a Biospheric Factor: A Course of Lectures), Yekaterinburg: Ural'sk. Gos. Tekh. Univ., 2007], favorably differs of those issues.

In the book, the author briefly (book volume about 7 author's quires) considers the contribution of radiation to the modern environmental situation. In the first three sections of the book, the author explains the most important notions, terms, and relationships pertaining to the radioactivity phenomenon and radiation safety. $\mathrm{He}$ also discusses the possible harmful effect of radiation on a human body and the ways of protection. Here it should be particularly emphasized that the author uses the terminology recommended by international organizations and accepted presently throughout the world. He also presents the modern norms concerning radiation safety. This is particularly important in view of the fact that in the past 10-15 years the dosimetric terminology and radiation safety regulations changed essentially.

Presentation in both the first and the subsequent sections of the book is accompanied by consideration of various radiation situations, primarily of those on in the Ural region. However, Egorov's analysis of environmental problems will be useful for readers from any region. The problems are discussed on a high scientific level, and at the same time the book is actually understandable by any reader with secondary education.

In the final section of the book, the author focuses attention on the fact that the arising problems with radiation safety of the population are frequently due not to objective reasons (regular operation of plants and installations) but to a "human factor," as it is usually formulated in mass media. In this case, the "human factor," as Egorov writes, consists in "insufficient general scientific and technical culture." Below he gives a more strongly-worded formulation: "The modern technosphere is... pitiless to amateurs and ignoramuses." We hope that publication of this book will favor a decrease in the number of both. Unfortunately, the printed number of copies is very few.

S. S. Berdonosov 